\title{
Optimal Operation of Distribution Networks Considering Energy Storage Devices
}

\author{
Leonardo H. Macedo, Student Member, IEEE, John F. Franco, Member, IEEE, Marcos J. Rider, Member, IEEE, \\ and Rubén Romero, Senior Member, IEEE
}

\begin{abstract}
This paper presents a mixed-integer second-order cone programing (MISOCP) model to solve the optimal operation problem of radial distribution networks (DNs) with energy storage. The control variables are the active and reactive generated power of dispatchable distributed generators (DGs), the number of switchable capacitor bank units in operation, the tap position of the voltage regulators and on-load tap-changers, and the operation state of the energy storage devices. The objective is to minimize the total cost of energy purchased from the distribution substation and the dispatchable DGs. The steady-state operation of the DN is modeled using linear and second-order cone programing. The use of an MISOCP model guarantees convergence to optimality using existing optimization software. A mixed-integer linear programing (MILP) formulation for the original model is also presented in order to show the accuracy of the proposed MISOCP model. An 11-node test system and a 42-node real system were used to demonstrate the effectiveness of the proposed MISOCP and MILP models.
\end{abstract}

Index Terms-Distributed generation, energy storage, mixed-integer linear programing (MILP), mixed-integer second-order cone programing (MISOCP), optimal operation of radial distribution networks, smart grid.

\section{ACRONYMS}

The abbreviations of common terms used in this paper are presented below.

$\begin{array}{ll}\text { CB } & \text { Fixed capacitor bank. } \\ \text { DG } & \text { Distributed generator. } \\ \text { DN } & \text { Distribution network. } \\ \text { DSS } & \text { Distribution substation. } \\ \text { ESD } & \text { Energy storage device. } \\ \text { MILP } & \text { Mixed-integer linear programing. } \\ \text { MINLP } & \text { Mixed-integer nonlinear programing. } \\ \text { MISOCP } & \text { Mixed-integer second-order cone programing. } \\ \text { OLTC } & \text { On-load tap-changer. } \\ \text { OODN } & \text { Optimal operation of DNs. } \\ \text { OPF } & \text { Optimal power flow. } \\ \text { RS } & \text { Renewable source. }\end{array}$

Manuscript received May 30, 2014; revised November 3, 2014 and February 9, 2015; accepted March 30, 2015. Date of publication April 27, 2015; date of current version October 17, 2015. This work was supported in part by the Conselho Nacional de Desenvolvimento Científico e Tecnológico under Grant 304234/2013-3, and in part by the Fundação de Amparo à Pesquisa do Estado de São Paulo under Grant 2012/23454-4.

The authors are with the Faculdade de Engenharia de Ilha Solteira, Departamento de Engenharia Elétrica, Universidade Estadual Paulista, Ilha Solteira 15385-000, São Paulo, Brazil (e-mail: mjrider@dee.feis.unesp.br).

Color versions of one or more of the figures in this paper are available online at http://ieeexplore.ieee.org.

Digital Object Identifier 10.1109/TSG.2015.2419134

\begin{abstract}
SCB Switchable capacitor bank
VR Voltage regulator.
\end{abstract}

\section{NOMENCLATURE}

The notation used throughout this paper is reproduced below for quick reference.

$\begin{array}{cl}\text { Sets } & \\ \Omega_{n} & \text { Set of nodes. } \\ \Omega_{b} & \text { Set of branches. } \\ \Omega_{d} & \text { Set of load levels. } \\ \Omega_{S} & \text { Set of DSS nodes. } \\ \Omega_{\mathrm{dg}} & \text { Set of dispatchable DGs. } \\ \Omega_{\mathrm{cb}} & \text { Set of CBs. } \\ \Omega_{\mathrm{scb}} & \text { Set of SCBs. } \\ \Omega_{\mathrm{vr}} & \text { Set of VRs. } \\ \Omega_{r s} & \text { Set of nondispatchable RSs. } \\ \Omega_{s d} & \text { Set of ESDs. }\end{array}$

\section{Function}

$f(y, \bar{y}, \Gamma)$ Approximation of the square value of $y$.

$\begin{gathered}\text { Constants } \\ c_{i, d}^{\mathrm{dg}}\end{gathered}$
$c_{d}^{S}$
$\Delta t$
$P_{i, d}^{D}$
$Q_{i, d}^{D}$
$R_{i j}$
$X_{i j}$
$Z_{i j}$
$\bar{I}_{i j}$
$\bar{V}$
$\frac{V}{V^{\mathrm{nom}}}$
$\bar{S}_{i}^{S}$
$\frac{p f}{i} \mathrm{dg}$
$\overline{p f}_{i}^{\mathrm{dg}}$

Purchase price of energy from dispatchable DG at node $i$ at load level $d$ (US\$/kWh).

Purchase price of energy from DSS at load level $d$ (US\$/kWh).

Time duration of each load level (h).

Active power load at node $i$ at load level $d(\mathrm{~kW})$.

Reactive power load at node $i$ at load level $d$ (kvar).

Resistance of branch ij $(m \Omega)$.

Reactance of branch ij $(m \Omega)$.

Impedance of branch $i j(m \Omega)$.

Maximum current magnitude of branch $i j$ (A).

Maximum voltage magnitude $(\mathrm{kV})$.

Minimum voltage magnitude $(\mathrm{kV})$.

Nominal voltage magnitude $(\mathrm{kV})$.

Maximum apparent power limit of substation at node $i(\mathrm{kVA})$.

Lower limit of the capacitive power factor for the DG at node $i$.

Lower limit of the inductive power factor for the DG at node $i$. 
$\bar{S}_{i}^{\mathrm{dg}} \quad$ Maximum apparent power limit of the DG at node $i$ (kVA).

$q_{i}^{\text {scb }} \quad$ Reactive power capacity of each module of the SCB at node $i$ (kvar).

$Q_{i}^{\text {cb }} \quad$ Reactive power injection of the $\mathrm{CB}$ at node $i$ (kvar).

$\bar{n}_{i, d}^{\mathrm{scb}} \quad$ Maximum integer number of SCB units at node $i$.

$\bar{\Delta}_{i}^{\mathrm{scb}}$

Maximum variation of capacitor bank units at node $i$ operating in the considered time period.

$r_{i j}^{\mathrm{vr}} \quad$ Regulation percentage of the VR connected to branch $i j$.

$\bar{l}_{i j}^{\mathrm{vr}} \quad$ Maximum number of steps of the VR in branch $i j$.

$\frac{\Delta_{i j}}{\mathrm{vr}}$

$P_{i, d}^{r s}$

$\underline{P}_{i}^{s d+}$

$\bar{P}_{i, d}^{s d+}$

$\underline{P}_{i}^{s d-}$

$\bar{P}_{i, d}^{s d-}$

$\underline{E}_{i}^{s d}$

$\bar{E}_{i}^{s d}$

$\eta_{i}^{s d+}$

$\eta_{i}^{s d-}$

$\frac{\beta_{i}^{s d}}{\Delta_{i}^{s d}}$

$\Gamma \quad$ Number of discretizations used in function $f$.

$\sigma_{y, \gamma} \quad$ Slope of the $\gamma$ th discretization of $y$.

$\bar{y} \quad$ Maximum value of $y$.

\section{Continuous Variables}

$V_{i, d}$

$V_{i, d}^{s q r}$

$P_{i, d}^{S}$

$Q_{i, d}^{S}$

$I_{i j, d}$

$I_{i j, d}^{s q r}$

$P_{i j, d}$

$Q_{i j, d}$

$P_{i j, d}^{\mathrm{vr}}$
Voltage magnitude at node $i$ at load level $d(\mathrm{kV})$. Square of $V_{i, d}$.

Active power supplied by substation at node $i$ at load level $d(\mathrm{~kW})$.

Reactive power supplied by substation at node $i$ at load level $d$ (kvar).

Current magnitude of branch $i j$ at load level $d$ (A).

Square of $I_{i j, d}$.

Active power flow of branch $i j$ at load level $d(\mathrm{~kW})$.

Reactive power flow of branch $i j$ at load level $d$ (kvar).

Active power flow of the VR between nodes $i$ and $j$ at load level $d(\mathrm{~kW})$.
$Q_{i j, d}^{\mathrm{vr}} \quad$ Reactive power flow of the VR between nodes $i$ and $j$ at load level $d$ (kvar).

$P_{i, d}^{\mathrm{dg}} \quad$ Active power generation of the DG at node $i$ at load level $d(\mathrm{~kW})$.

$Q_{i, d}^{\mathrm{dg}} \quad$ Reactive power generation of the DG at node $i$ at load level $d$ (kvar).

$Q_{i, d}^{\mathrm{scb}} \quad$ Reactive power injection of the SCB at node $i$ at load level $d$ (kvar).

$t_{i j, d}^{\mathrm{vr}} \quad$ Tap of the VR in branch $i j$ at load level $d$.

$V_{i, j, d, k}^{c}$ Correction variable used in the linearization of the VR model.

$P_{i, d}^{s d+} \quad$ Active power production (injection) of the storage device at node $i$ at load level $d(\mathrm{~kW})$.

$P_{i, d}^{s d-} \quad$ Active power storage (extraction) of the storage device at node $i$ at load level $d(\mathrm{~kW})$.

$E_{i, d}^{s d} \quad$ State of charge of the storage device at node $i$ at load level $d(\mathrm{kWh})$.

$n_{i, d}^{+} \quad$ Auxiliary variable that indicates a positive change in the number of SCB units connected at node $i$.

$n_{i, d}^{-} \quad$ Auxiliary variable that indicates a negative change in the number of SCB units connected at node $i$.

$l_{i j, d}^{+} \quad$ Auxiliary variable that indicates a positive variation in the steps of the tap of the VR in branch $i j$.

$l_{i j, d}^{-} \quad$ Auxiliary variable that indicates a negative variation in the steps of the tap of the VR in branch $i j$.

$e_{i, d}^{+} \quad$ Auxiliary variable that indicates a positive change in the operation state of the storage device at node $i$.

$e_{i, d}^{-} \quad$ Auxiliary variable that indicates a negative change in the operation state of the storage device at node $i$.

$\delta_{y, \gamma} \quad$ Value of the $\gamma$ th auxiliary variable used in the discretization of $y$.

$y \quad$ Argument of function $f$.

$y^{+} \quad$ Auxiliary variable in the calculation of $|y|$.

$y^{-} \quad$ Auxiliary variable in the calculation of $|y|$.

\section{Binary Variables}

$e_{i, d}^{s d} \quad$ Operation state of the storage device at node $i$.

$b_{i j, d, k}^{\mathrm{vr}} \quad$ Binary variable that defines the operation region for the tap of the VR in branch $i j$.

\section{Integer Variables}

$n_{i, d}^{\mathrm{scb}} \quad$ Number of SCB units in operation at node $i$ at load level $d$.

$l_{i j, d}^{\mathrm{vr}} \quad$ Number of tap steps of the VR connected to branch $i j$ at load level $d$.

\section{INTRODUCTION}

$\mathbf{T}$ HE PROBLEM of the OODN considering ESDs aims at minimizing an objective function by determining the optimal values for a set of decision variables. The decision variables for the OODN problem are commonly the tap 
position in the OLTCs and VRs, the number of units in operation in each SCB, the active and reactive power injections by dispatchable DGs, and the operation state (injection or extraction of power from the network) of the ESDs. The objective is to minimize the total purchase cost of energy from the DSS and the dispatchable DGs over a predetermined period of time [1].

In the specialized literature, works dealing with the OODN problem have only considered some of the devices previously cited [2]-[7]; moreover, these works have not addressed energy storage. Reference [2] presented a genetic algorithm (GA) to obtain the optimal control of voltage magnitude in DNs with VRs, SCBs, DGs, and static var compensators. Reference [3] proposed an evolutionary algorithm to solve the OODN problem, accounting for SCBs, OLTCs in the substation, and the reconfiguration of the system. In [4], VRs, OLTCs, and photovoltaic generation were considered with the objective of minimizing the number of tap operations of the VRs, employing an optimal coordination strategy. A model for the OODN problem was proposed in [5] with the goal of minimizing power losses; in addition, this model included photovoltaic generation and reconfiguration of the system. Reference [6] presented an MILP model for the OODN problem considering VRs, SCBs, and DGs. Reference [7] proposed a Chu-Beasley's GA and a simplified algorithm to solve the OODN problem, also taking VRs, SCBs, and DGs into account.

In [8]-[12], the optimal dispatch of ESDs was examined, but with respect to simple DN topologies. Reference [13] listed many benefits of using ESDs in DNs. Despite all of these benefits, energy storage is still expensive for high penetrations. However, with the development of technology, the price of ESDs has been decreasing and cost/benefit analyses have shown that the application of these devices is justified in some cases [13].

There have also been works tackling RSs and ESDs simultaneously, along with the general network topologies in the OODN problem [14]-[18]. However, due to the complexity of the model, VRs, SCBs, and DGs have not been dealt with. For example, Levron et al. [14] presented a methodology for solving the OPF in microgrids with energy storage and RSs. The objective was to minimize the energy purchase cost at the point of common coupling. The proposed method used a load flow and dynamic search algorithm to attempt in finding an optimal solution for the OODN problem. The method worked well for systems with a small number of ESDs, but could not solve problems with more than five ESDs. Furthermore, the proposed methodology did not guarantee convergence to a global optimum.

Reference [15] explicated a solution for active-reactive OPF in DNs with RSs and ESDs. Although both injected and extracted power were flexible, i.e., they could assume different values, the charging and discharging periods were fixed during the days - a consideration that led to low-quality solutions. Reference [16] made an improvement to the model in [15] by incorporating the flexible operation of the ESDs, i.e., both injected and extracted power by ESDs, and by allowing the duration of the injection and extraction of

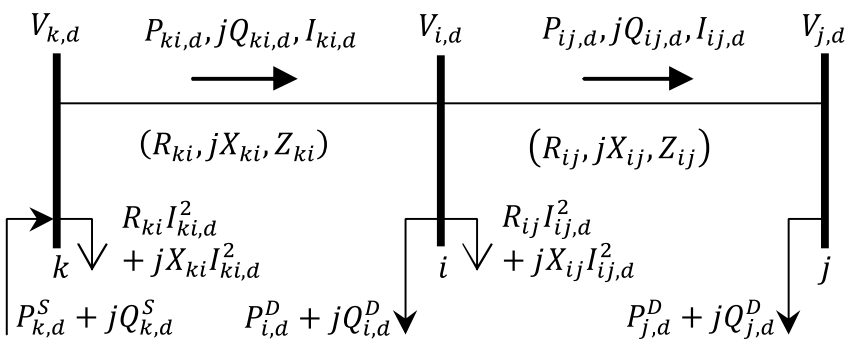

Fig. 1. Illustrative DN with three nodes.

energy from the network to vary. The methodologies presented in [15] and [16] are applicable to meshed networks. The solution technique proposed in [16] assumes only one charge/discharge cycle per day.

Reference [17] proposed a dynamic OPF to solve the OODN considering only RSs, energy storage, and active network management. Reference [18] presented several models with which to formulate ideal and generic storage devices. Most of these works [2]-[17] used metaheuristic or heuristic techniques to solve the OODN problem. Although these techniques are robust, flexible, and achieve good results, they present many problems, such as a high computational demand, the adjustment and tuning of parameters, and the definition of a stop criterion. In addition, they cannot guarantee convergence to the global optimum [19]. Also, [14]-[18] did not consider the presence of VRs, SCBs, and dispatchable DGs in the DN.

This paper presents an MISOCP model for solving the OODN problem, guaranteeing convergence to the global optimum of the original MINLP model. The proposed model considers dispatchable DGs, SCBs, VRs, OLTCs, RSs, and ESDs simultaneously and is applicable to radial networks in general. In addition, the proposed formulation for ESDs permits flexible operation and modification to the number of charge cycles in the period of analysis. The use of an MISOCP model guarantees optimality by using existing classical optimization tools. An approximated MILP model is also presented in order to show the accuracy of the proposed MISOCP model. The main contribution of this paper is a novel MISOCP model for solving the OODN problem considering ESDs, which has the following benefits: 1) a flexible, realistic, and precise model; 2) efficient computational behavior with conventional MISOCP solvers; and 3) convergence to optimality that is guaranteed by using classical optimization techniques.

\section{MOdELS FOR THE OODN PROBLEM}

\section{A. Steady-State Operation of Distribution Networks}

The following assumptions are made in order to represent the steady-state operation of a radial DN.

1) The load is represented as constant active and reactive power.

2) The active and reactive power losses of branch $i j$ are concentrated at node $i$.

3) The DN is balanced and represented by a single-phase equivalent.

These three considerations are shown in Fig. 1 for each load level $d$. The terms $R_{i j} I_{i j, d}^{2}$ and $X_{i j} I_{i j, d}^{2}$ represent the active 

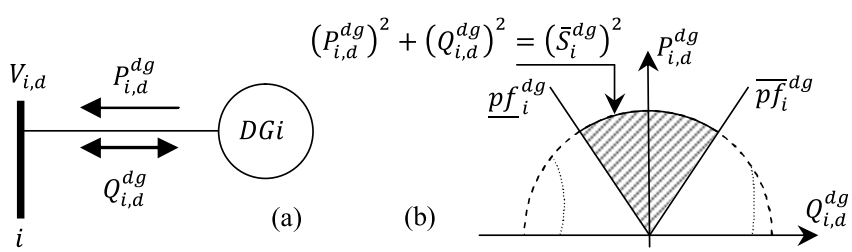

Fig. 2. Dispatchable DG. (a) Scheme and (b) capability curve.

and reactive power losses of branch $i j$, respectively. The equations representing the steady-state operation of a radial DN are shown in (1)-(4) [20]. These equations are frequently used in the load flow sweep method [21], [22]

$$
\begin{aligned}
\sum_{k i \in \Omega_{b}} P_{k i, d}-\sum_{i j \in \Omega_{b}}\left(P_{i j, d}+R_{i j} I_{i j, d}^{2}\right)+ & P_{i, d}^{S}=P_{i, d}^{D} \\
& \forall i \in \Omega_{n}, \forall d \in \Omega_{d} \\
\sum_{k i \in \Omega_{b}} Q_{k i, d}-\sum_{i j \in \Omega_{b}}\left(Q_{i j, d}+X_{i j} I_{i j, d}^{2}\right)+ & Q_{i, d}^{S}=Q_{i, d}^{D} \\
& \forall i \in \Omega_{n}, \forall d \in \Omega_{d} \\
V_{i, d}^{2}-V_{j, d}^{2}=2\left(R_{i j} P_{i j, d}+X_{i j} Q_{i j, d}\right)+ & Z_{i j}^{2} I_{i j, d}^{2} \\
& \forall i j \in \Omega_{b}, \forall d \in \Omega_{d} \\
I_{i j, d}^{2} V_{j, d}^{2}=P_{i j, d}^{2}+Q_{i j, d}^{2} & \forall i j \in \Omega_{b}, \forall d \in \Omega_{d} .
\end{aligned}
$$

The active and reactive power balance equations are represented by (1) and (2), respectively. The magnitude of voltage drop is calculated in terms of the active and reactive power flows, the current magnitude, and the electrical parameters of branch $i j$, as expressed in (3), while (4) is the square current magnitude calculation.

\section{B. Modeling of the Equipment}

1) Dispatchable Distributed Generators: In this paper, it is assumed that dispatchable DGs (e.g., biomass generators) are synchronous machines that are sources of both active and reactive power. It is also assumed that DGs operate with a restricted power factor and free terminal voltage. Fig. 2(a) shows the scheme of a dispatchable DG and Fig. 2(b) shows its capability curve. Equations (5)-(7) model the operation of dispatchable DGs

$$
\begin{aligned}
& \left(P_{i, d}^{\mathrm{dg}}\right)^{2}+\left(Q_{i, d}^{\mathrm{dg}}\right)^{2} \leq\left(\bar{S}_{i}^{\mathrm{dg}}\right)^{2} \quad \forall i \in \Omega_{\mathrm{dg}}, \forall d \in \Omega_{d} \\
& P_{i, d}^{\mathrm{dg}} \geq 0 \\
& \quad \forall i \in \Omega_{\mathrm{dg}}, \forall d \in \Omega_{d} \\
& -P_{i, d}^{\mathrm{dg}} \tan \left(\cos ^{-1}\left(\underline{p f}_{i}^{\mathrm{dg}}\right)\right) \leq Q_{i, d}^{\mathrm{dg}} \leq P_{i, d}^{\mathrm{dg}} \tan \left(\cos ^{-1}\left(\overline{p f}_{i}^{\mathrm{dg}}\right)\right) \\
& \forall i \in \Omega_{\mathrm{dg}}, \forall d \in \Omega_{d} .
\end{aligned}
$$

Constraint (5) is the power generation capacity, while (6) establishes that the active power generated by the dispatchable DG connected at node $i$ is always positive. Constraint (7) limits the reactive power generation to within capacitive and inductive minimum power factors.

2) Capacitor Banks: There are two types of capacitor banks: 1) CBs and 2) SCBs. CBs are formed by units that are always connected to the DN, whereas SCBs have units that may be fully or partially connected to the DN. Fig. 3 shows

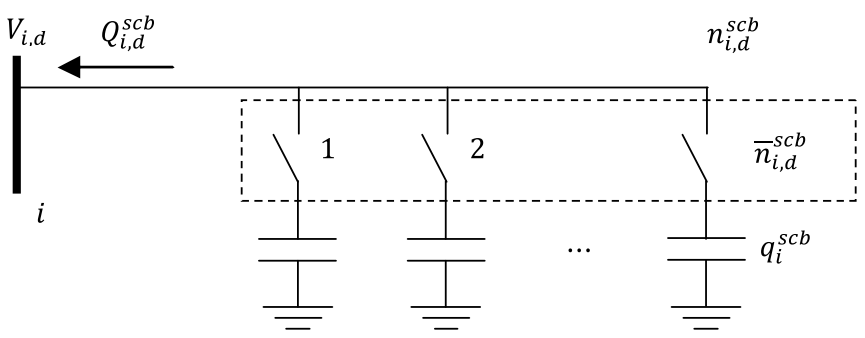

Fig. 3. SCB.

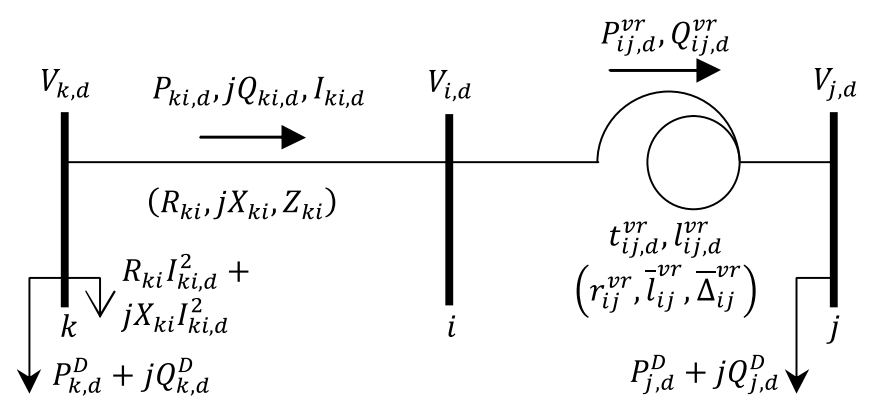

Fig. 4. VR.

a scheme of an SCB. Expressions (8)-(11) model the operation of SCBs

$Q_{i, d}^{\mathrm{scb}}=n_{i, d}^{\mathrm{scb}} q_{i}^{\mathrm{scb}}$

$\sum_{d \in \Omega_{d}}\left|n_{i, d}^{\mathrm{scb}}-n_{i, d-1}^{\mathrm{scb}}\right| \leq \bar{\Delta}_{i}^{\mathrm{scb}} \quad \forall i \in \Omega_{\mathrm{scb}}$

$0 \leq n_{i, d}^{\mathrm{scb}} \leq \bar{n}_{i, d}^{\mathrm{scb}}$

$\forall i \in \Omega_{\mathrm{scb}}, \forall d \in \Omega_{d}$

$n_{i, d}^{\mathrm{scb}}$ integer

$\forall i \in \Omega_{\mathrm{scb}}, \forall d \in \Omega_{d}$.

Equation (8) imposes that the total reactive power injected by the SCB connected at node $i$ at load level $d$ is equal to the number of units connected at the node times the reactive power of each unit. Constraint (9) limits the number of changes in the operation of switchable units during the period of analysis, where for $d=0, n_{i, 0}^{\mathrm{scb}}$ is the initial value of $n_{i, d}^{\mathrm{scb}} ;(10)$ limits the number of units connected at a node. The integer condition of the number of units connected is established by (11).

3) Voltage Regulators and OLTCs: VRs and OLTCs aim at improving voltage levels in DNs. They have a mechanism that allows the adjustment of the tap, thus adjusting the value of regulation. In this paper, the switching mechanisms of VRs and OLTCs are remotely controlled. Fig. 4 shows a VR connected between nodes $i$ and $j$. Equations (12)-(16) show the model of the VR

$$
\begin{array}{ll}
V_{j, d}=t_{i j, d}^{\mathrm{vr}} V_{i, d} & \forall i j \in \Omega_{\mathrm{vr}}, \forall d \in \Omega_{d} \\
t_{i j, d}^{\mathrm{vr}}=1+r_{i j}^{\mathrm{vr}} l_{i j, d}^{\mathrm{vr}} & \forall i j \in \Omega_{\mathrm{vr}}, \forall d \in \Omega_{d} \\
\sum_{d \in \Omega_{d}}\left|l_{i j, d}^{\mathrm{vr}}-l_{i j, d-1}^{\mathrm{vr}}\right| \leq \bar{\Delta}_{i j}^{\mathrm{vr}} & \forall i j \in \Omega_{\mathrm{vr}} \\
-\bar{l}_{i j}^{\mathrm{vr}} \leq l_{i j, d}^{\mathrm{vr}} \leq \bar{l}_{i j}^{\mathrm{vr}} & \forall i j \in \Omega_{\mathrm{vr}}, \forall d \in \Omega_{d} \\
l_{i j, d}^{\mathrm{vr}} \text { integer } & \forall i j \in \Omega_{\mathrm{vr}}, \forall d \in \Omega_{d} .
\end{array}
$$

Equation (12) calculates the voltage at node $j$ as a function of the nonregulated voltage at node $i$ and the value of 


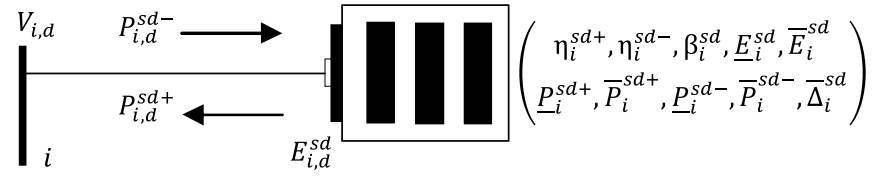

Fig. 5. ESD

the tap. From (13), it can be shown that the value of the tap varies in the range of regulation $\left[1-r_{i j}^{\mathrm{vr}}, 1+r_{i j}^{\mathrm{vr}}\right]$ in intervals of $r_{i j}^{\mathrm{vr}} / \bar{l}_{i j}^{\mathrm{vr}}$. Constraint (14) defines the maximum variation of the VR/OLTC tap steps during the period of analysis, where for $d=0, l_{i j, 0}^{\mathrm{vr}}$ is the initial value of $l_{i j, d}^{\mathrm{vr}}$. Expression (15) represents the interval of variation of the discrete steps of the tap. Expression (16) determines that the steps of the VRs and OLTCs must be integers [6].

4) Intermittent Renewable Sources: Intermittent RSs of energy (wind turbines and photovoltaic panels) are considered in this paper as nondispatchable sources operating with unity power factor, i.e., they can only produce active power. Because they are nondispatchable, they are represented as injections of active power in the node at which they are installed.

Thus, the value of the injected power is a parameter, and not a variable, that depends on the weather forecast, as shown in [14].

5) Energy Storage Devices: It is appropriate to store energy in storage devices in two situations: 1) when energy from nondispatchable RSs is in excess in the system and 2) when the purchase costs of energy from DSS or dispatchable DGs are low. The stored energy can then be consumed during peak hours, when energy is expensive and load is high [10]. Fig. 5 shows a diagram of the model of an ESD connected at node $i$. Equations (17)-(22) model the operation of the ESDs

$$
\begin{array}{lc}
\underline{P}_{i}^{s d+} e_{i, d}^{s d} \leq P_{i, d}^{s d+} \leq \bar{P}_{i, d}^{s d+} e_{i, d}^{s d} & \forall i \in \Omega_{s d}, \forall d \in \Omega_{d} \\
\underline{P}_{i}^{s d-}\left(1-e_{i, d}^{s d}\right) \leq P_{i, d}^{s d-} \leq \bar{P}_{i, d}^{s d-}\left(\begin{array}{r}
1-e_{i, d}^{s d} \\
\forall i \in \Omega_{s d}, \forall d \in \Omega_{d} \\
\forall i, P_{i, d}^{s d}-\beta_{i}^{s d} \Delta t E_{i, d}^{s d}
\end{array}\right. \\
\begin{array}{l}
E_{i, d}^{s d}=E_{i, d-1}^{s d}+\eta_{i}^{s d-} \Delta t P_{i, d}^{s d-}-\frac{1}{\eta_{i}^{s d+}} \Delta t P_{i, d}^{s d+} \\
\forall i \in \Omega_{s d}, \forall d \in \Omega_{d}
\end{array} \\
\underline{E}_{i}^{s d} \leq E_{i, d}^{s d} \leq \bar{E}_{i}^{s d} & \forall i \in \Omega_{s d}, \forall d \in \Omega_{d} \\
\sum_{d \in \Omega_{d}}\left|e_{i, d}^{s d}-e_{i, d-1}^{s d}\right| \leq \bar{\Delta}_{i j}^{s d} & \forall i \in \Omega_{s d} \\
e_{i, d}^{s d} \in\{0,1\} & \forall i \in \Omega_{s d}, \forall d \in \Omega_{d}
\end{array}
$$

where if $e_{i, d}^{s d}=0$, the ESD is extracting power from the DN and if $e_{i, d}^{s d}=1$, the ESD is injecting power into the DN. Constraint (17) limits power injection and (18) restricts power extraction from the ESDs. Equation (19) determines that the stored energy (charge state) of the ESD connected at node $i$ at load level $d$ depends on the previous state of charge, the injected and extracted power for the time interval (duration of load level) multiplied by their respective efficiencies, and the self-discharge rate of the ESD. For $d=0, E_{i, 0}^{s d}$ is the initial value of $E_{i, d}^{s d}$. Expression (20) represents the extent of the charge state, i.e., the maximum and minimum energy that must remain in the ESD. Equation (21), together with (17) and (18), limits the number of changes in the operation state of the ESDs during the period of analysis, where for $d=0, e_{i, 0}^{s d}$ is the initial value of $e_{i, d}^{s d}$. Equation (22) imposes that $e_{i, d}^{s d}$ must be binary.

6) Additional Operational Constraints: Constraints (23)-(25) are also part of the model

$$
\begin{aligned}
\left(P_{i, d}^{S}\right)^{2}+\left(Q_{i, d}^{S}\right)^{2} & \leq\left(\bar{S}_{i}^{S}\right)^{2} & & \forall i \in \Omega_{S}, \forall d \in \Omega_{d} \\
\underline{V} \leq V_{i, d} & \leq \bar{V} & & \forall i \in \Omega_{n}, \forall d \in \Omega_{d} \\
0 \leq I_{i j, d} & \leq \bar{I}_{i j} & & \forall i j \in \Omega_{b}, \forall d \in \Omega_{d} .
\end{aligned}
$$

Constraint (23) models the generation capacity of the substation, (24) defines the voltage limits of the nodes, and (25) confines the current in the conductors to its maximum supported current.

\section{Mixed-Integer Nonlinear Programing Model}

The objective in this paper is to minimize the energy purchase cost of the DSS and dispatchable DGs, as shown in (26). Equations (27) and (28) are obtained by adding the contributions of all of the devices cited previously to the active and reactive power balance equations (1) and (2). The model for the OODN problem is described

$$
\min \sum_{d \in \Omega_{d}} \sum_{i \in \Omega_{S}} c_{d}^{S} \Delta t P_{i, d}^{S}+\sum_{d \in \Omega_{d}} \sum_{i \in \Omega_{\mathrm{dg}}} c_{d}^{\mathrm{dg}} \Delta t P_{i, d}^{\mathrm{dg}}
$$

subject to constraints (3)-(25)

$$
\begin{gathered}
\sum_{k i \in \Omega_{b}} P_{k i, d}-\sum_{i j \in \Omega_{b}}\left(P_{i j, d}+R_{i j} I_{i j, d}^{2}\right)+\sum_{k i \in \Omega_{\mathrm{vr}}} P_{k i, d}^{\mathrm{vr}}-\sum_{i j \in \Omega_{\mathrm{vr}}} P_{i j, d}^{\mathrm{vr}} \\
+P_{i, d}^{S}+P_{i, d}^{\mathrm{dg}}+P_{i, d}^{r s}+P_{i, d}^{s d+}-P_{i, d}^{s d-}=P_{i, d}^{D} \\
\forall i \in \Omega_{n}, \forall d \in \Omega_{d} \\
\sum_{k i \in \Omega_{b}} Q_{k i, d}-\sum_{i j \in \Omega_{b}}\left(Q_{i j, d}+X_{i j} I_{i j, d}^{2}\right)+\sum_{k i \in \Omega_{\mathrm{vr}}} Q_{k i, d}^{\mathrm{vr}}-\sum_{i j \in \Omega_{\mathrm{vr}}} Q_{i j, d}^{\mathrm{vr}} \\
+Q_{i, d}^{S}+Q_{i, d}^{\mathrm{dg}}+Q_{i}^{\mathrm{cb}}+Q_{i, d}^{\mathrm{scb}}=Q_{i, d}^{D} \\
\forall i \in \Omega_{n}, \forall d \in \Omega_{d} .
\end{gathered}
$$

The proposed model (26)-(28) is an MINLP problem due to the following: (3)-(5), (23), (27), and (28) have squared variables and the product of squared variables; (12) presents the product of two variables; moreover, (9), (14), and (21) are nonlinear because they present the sum of the absolute value of a difference in variables. The presence of integer and binary variables makes this problem even more difficult to solve. Existing methodologies are able to find only low-quality solutions, and convergence, even for a feasible solution, is not guaranteed.

\section{Mixed-Integer Second-Order Cone Programing Model}

This section presents an MISOCP model for the OODN problem considering ESDs. The process to obtain this model from (26) to (28) involves variable substitutions, convexification of constraints [23], and an equivalent disjunctive formulation for the model of VRs and OLTCs. In (3), (4), (27), and (28), the voltage and current magnitudes 
are squared, so that the change in variables can be accomplished

$$
\begin{aligned}
V_{i, d}^{s q r} & =V_{i, d}^{2} \\
I_{i j, d}^{s q r} & =I_{i j, d}^{2} .
\end{aligned}
$$

After this, (3), (27), and (28) become linear

$$
\begin{gathered}
\sum_{k i \in \Omega_{b}} P_{k i, d}-\sum_{i j \in \Omega_{b}}\left(P_{i j, d}+R_{i j} I_{i j, d}^{s q r}\right)+\sum_{k i \in \Omega_{\mathrm{vr}}} P_{k i, d}^{\mathrm{vr}} \\
-\sum_{i j \in \Omega_{\mathrm{vr}}} P_{i j, d}^{\mathrm{vr}}+P_{i, d}^{S}+P_{i, d}^{\mathrm{dg}}+P_{i, d}^{r s}+P_{i, d}^{s d+}-P_{i, d}^{s d-}=P_{i, d}^{D} \\
\forall i \in \Omega_{n}, \forall d \in \Omega_{d} \\
\sum_{k i \in \Omega_{b}} Q_{k i, d}-\sum_{i j \in \Omega_{b}}\left(Q_{i j, d}+X_{i j} I_{i j, d}^{s q r}\right)+\sum_{k i \in \Omega_{\mathrm{vr}}} Q_{k i, d}^{\mathrm{vr}} \\
-\sum_{i j \in \Omega_{\mathrm{vr}}} Q_{i j, d}^{\mathrm{vr}}+Q_{i, d}^{S}+Q_{i, d}^{\mathrm{dg}}+Q_{i}^{\mathrm{cb}}+Q_{i, d}^{\mathrm{scb}}=Q_{i, d}^{D} \\
\forall i \in \Omega_{n}, \forall d \in \Omega_{d} \\
V_{i, d}^{s q r}-V_{j, d}^{s q r}=2\left(R_{i j} P_{i j, d}+X_{i j} Q_{i j, d}\right)+Z_{i j}^{2} I_{i j, d}^{s q r} \\
\forall i j \in \Omega_{b}, \forall d \in \Omega_{d} .
\end{gathered}
$$

Additionally, (24) and (25) are expressed in a linear form

$$
\begin{aligned}
\underline{V}^{2} \leq V_{i, d}^{s q r} \leq \bar{V}^{2} & \forall i \in \Omega_{n}, \forall d \in \Omega_{d} \\
0 \leq I_{i j, d}^{s q r} \leq \bar{I}_{i j}^{2} & \forall i j \in \Omega_{b}, \forall d \in \Omega_{d}
\end{aligned}
$$

Conversely, (4) continues to be nonlinear because on its lefthand side there is a product of variables and on its right-hand side there are squared variables

$$
I_{i j, d}^{s q r} V_{j, d}^{s q r}=P_{i j, d}^{2}+Q_{i j, d}^{2} \quad \forall i j \in \Omega_{b}, \forall d \in \Omega_{d} .
$$

However, convexity and optimality can be achieved by relaxing the equalities in (36) into the second-order conic constraints

$$
I_{i j, d}^{s q r} V_{j, d}^{s q r} \geq P_{i j, d}^{2}+Q_{i j, d}^{2} \quad \forall i j \in \Omega_{b}, \forall d \in \Omega_{d}
$$

If the dual variables related to constraints (37) in the OODN problem are greater than zero, then the conic constraint (37) is active and, therefore, is equivalent to the nonlinear constraint (36) [24]. A set of sufficient conditions for constraint (37) to be active is provided in [25].

As a result of the variable changes (29) and (30), (12) is squared on both sides, and the squared value of the tap is needed in (13). Also, (12) presents a product of two variables: one continuous and one integer. Expressions (12) and (13) are (38) and (39) after altering the variable

$$
\begin{aligned}
& V_{j, d}^{s q r}=t_{i j, d}^{s q r} V_{i, d}^{s q r} \forall i j \in \Omega_{\mathrm{vr}}, \forall d \in \Omega_{d} \\
& t_{i j, d}^{s q r}=1+2 r_{i j}^{\mathrm{vr}} \frac{l_{i j, d}^{\mathrm{vr}}}{\bar{l}_{i j}^{\mathrm{vr}}}+\left(r_{i j}^{\mathrm{vr}}\right)^{2}\left(\frac{l_{i j, d}^{\mathrm{vr}}}{\bar{l}_{i j}^{\mathrm{vr}}}\right)^{2} \\
& \forall i j \in \Omega_{\mathrm{vr}}, \forall d \in \Omega_{d} .
\end{aligned}
$$

The integer variable $l_{i j, d}^{\mathrm{vr}}$ can be represented by a set of binary variables

$$
\begin{array}{ll}
l_{i j, d}^{\mathrm{vr}}=\sum_{k=0}^{2 \bar{l}_{i j}^{\mathrm{vr}}}\left[\left(k-\bar{l}_{i j}^{\mathrm{vr}}\right) b_{i j, d, k}^{\mathrm{vr}}\right] \quad \forall i j \in \Omega_{\mathrm{vr}}, \forall d \in \Omega_{d} \\
\sum_{k=0}^{2 l_{i j}^{\mathrm{vr}}} b_{i j, d, k}^{\mathrm{vr}}=1 \\
b_{i j, d, k}^{\mathrm{vr}} \in\{0,1\} \quad \forall i j \in \Omega_{\mathrm{vr}}, \forall d \in \Omega_{d}, \forall k=0 \ldots 2 \bar{l}_{i j}^{\mathrm{vr}} .
\end{array}
$$

Using the representation in (40)-(42), the maximum variation of taps during the analyzed period (14) is modeled

$$
\sum_{d \in \Omega_{d}}\left|\sum_{k=0}^{2 \bar{l}_{i j}^{\mathrm{vr}}}\left[\left(k-\bar{l}_{i j}^{\mathrm{vr}}\right) b_{i j, d, k}^{\mathrm{vr}}\right]-\sum_{k=0}^{2 \bar{l}_{i j}^{\mathrm{vr}}}\left[\left(k-\bar{l}_{i j}^{\mathrm{vr}}\right) b_{i j, d-1, k}^{\mathrm{vr}}\right]\right| \leq \bar{\Delta}_{i j}^{\mathrm{vr}}
$$

The squared value of the tap is shown

$$
t_{i j, d}^{s q r}=\sum_{k=0}^{2 \bar{l}_{i j}^{\mathrm{vr}}}\left[\left(1+r_{i j}^{\mathrm{vr}} \frac{\left(k-\bar{l}_{i j}^{\mathrm{vr}}\right)}{\bar{l}_{i j}^{\mathrm{vr}}}\right)^{2} b_{i j, d, k}^{\mathrm{vr}}\right]_{\forall i j \in \Omega_{\mathrm{vr}}, \forall d \in \Omega_{d} .}
$$

The squared value of the regulated voltage can be calculated

$V_{j, d}^{s q r}=\sum_{k=0}^{2 \bar{l}_{i j}^{\mathrm{vr}}}\left[\left(1+r_{i j}^{\mathrm{vr}} \frac{\left(k-\bar{l}_{i j}^{\mathrm{vr}}\right)}{\bar{l}_{i j}^{\mathrm{vr}}}\right)^{2} V_{i, d}^{s q r} b_{i j, d, k}^{\mathrm{vr}}\right]$

$$
\forall i j \in \Omega_{\mathrm{vr}}, \forall d \in \Omega_{d} .
$$

The nonlinear product $V_{i, d}^{s q r} b_{i j, d, k}^{\mathrm{vr}}$ is represented by using the variable $V_{i, j, d, k}^{c}$

$$
\begin{array}{r}
V_{j, d}^{s q r}=\sum_{k=0}^{2 \bar{l}_{i j}^{\mathrm{vr}}}\left[\left(1+r_{i j}^{\mathrm{vr}} \frac{\left(k-\bar{l}_{i j}^{\mathrm{vr}}\right)}{\bar{l}_{i j}^{\mathrm{vr}}}\right)^{2} V_{i, j, d, k}^{c}\right] \\
\forall i j \in \Omega_{\mathrm{vr}}, \forall d \in \Omega_{d} . \\
V_{i, j, d, k}^{c}=V_{i, d}^{s q r} b_{i j, d, k}^{\mathrm{vr}} \quad \forall i j \in \Omega_{\mathrm{vr}}, \forall d \in \Omega_{d}, \forall k=0 \ldots 2 \bar{l}_{i j}^{\mathrm{vr}} .
\end{array}
$$

Equation (46) is linear, but (47) presents the product of a continuous variable and a binary variable that can be substituted by two equivalent linear constraints, according to the disjunctive formulation shown

$$
\begin{aligned}
& \begin{array}{l}
\underline{V}^{2} b_{i j, d, k}^{\mathrm{vr}} \leq V_{i, j, d, k}^{c} \leq \bar{V}^{2} b_{i j, d, k}^{\mathrm{vr}} \\
\forall i j \in \Omega_{\mathrm{vr}}, \forall d \in \Omega_{d}, \forall k=0 \ldots 2 \bar{l}_{i j}^{\mathrm{vr}} \\
\underline{V}^{2}\left(1-b_{i j, d, k}^{\mathrm{vr}}\right) \leq V_{i, d}^{\mathrm{sqr}}-V_{i, j, d, k}^{c} \leq \bar{V}^{2}\left(1-b_{i j, d, k}^{\mathrm{vr}}\right) \\
\forall i j \in \Omega_{\mathrm{vr}}, \forall d \in \Omega_{d}, \forall k=0 \ldots 2 \bar{l}_{i j}^{\mathrm{vr}} \\
b_{i j, d, k}^{\mathrm{vr}} \in\{0,1\} \quad \forall i j \in \Omega_{\mathrm{vr}}, \forall d \in \Omega_{d}, \forall k=0 \ldots 2 \bar{l}_{i j}^{\mathrm{vr}} .
\end{array}
\end{aligned}
$$

After all of the aforementioned changes, the model still presents three constraints that are nonlinear, i.e. (9), (14) and (21). However, note that (14) was replaced by (43). These constraints limit the number of changes in 
operation of the SCBs, VRs, and ESDs, respectively, during the period of analysis.

A linear equivalent to constraint (9) is shown

$$
\begin{array}{ll}
\sum_{d \in \Omega_{d}}\left(n_{i, d}^{+}+n_{i, d}^{-}\right) \leq \bar{\Delta}_{i}^{\mathrm{scb}} & \forall i \in \Omega_{\mathrm{scb}} \\
n_{i, d}^{\mathrm{scb}}-n_{i, d-1}^{\mathrm{scb}}=n_{i, d}^{+}-n_{i, d}^{-} & \forall i \in \Omega_{\mathrm{scb}}, \forall d \in \Omega_{d} \\
n_{i, d}^{+} \geq 0 & \forall i \in \Omega_{\mathrm{scb}}, \forall d \in \Omega_{d} \\
n_{i, d}^{-} \geq 0 & \forall i \in \Omega_{\mathrm{scb}}, \forall d \in \Omega_{d} .
\end{array}
$$

A similar linear equivalent can be obtained in (43). Constraints (55)-(58) represent a linear equivalent to (43)

$$
\begin{array}{ll}
\sum_{d \in \Omega_{d}}\left(l_{i j, d}^{+}+l_{i j, d}^{-}\right) \leq \bar{\Delta}_{i j}^{\mathrm{vr}} & \forall i j \in \Omega_{\mathrm{vr}} \\
\sum_{k=0}^{2 l_{i j}^{\mathrm{vr}}}\left[\left(k-\bar{l}_{i j}^{\mathrm{vr}}\right) b_{i j, d, k}^{\mathrm{vr}}\right]-\sum_{k=0}^{2 l_{i j}^{\mathrm{vr}}}[ & \left.\left(k-\bar{l}_{i j}^{\mathrm{vr}}\right) b_{i j, d-1, k}^{\mathrm{vr}}\right]=l_{i j, d}^{+}-l_{i j, d}^{-} \\
l_{i j, d}^{+} \geq 0 & \forall i j \in \Omega_{\mathrm{vr}}, \forall d \in \Omega_{d} \\
l_{i j, d}^{-} \geq 0 & \forall i j \in \Omega_{\mathrm{vr}}, \forall d \in \Omega_{d} \\
\forall i j \in \Omega_{\mathrm{vr}}, \forall d \in \Omega_{d}
\end{array}
$$

For constraint (21), the linear equivalent is

$$
\begin{aligned}
\sum_{d \in \Omega_{d}}\left(e_{i, d}^{+}+e_{i, d}^{-}\right) & \leq \bar{\Delta}_{i}^{s d} & & \forall i \in \Omega_{s d} \\
e_{i, d}^{s d}-e_{i, d-1}^{s d} & =e_{i, d}^{+}-e_{i, d}^{-} & & \forall i \in \Omega_{s d}, \forall d \in \Omega_{d} \\
0 \leq e_{i, d}^{+} & \leq 1 & & \forall i \in \Omega_{s d}, \forall d \in \Omega_{d} \\
0 \leq e_{i, d}^{-} & \leq 1 & & \forall i \in \Omega_{s d}, \forall d \in \Omega_{d} .
\end{aligned}
$$

Now, after these substitutions and linearizations, it is possible to write an MISOCP model for solving the OODN problem considering ESDs

$$
\min \sum_{d \in \Omega_{d}} \sum_{i \in \Omega_{S}} c_{d}^{S} \Delta t P_{i, d}^{S}+\sum_{d \in \Omega_{d}} \sum_{i \in \Omega_{\mathrm{dg}}} c_{d}^{\mathrm{dg}} \Delta t P_{i, d}^{\mathrm{dg}}
$$

subject to (5)-(7), (8), (10), (11), (17)-(20), (22), (23),

$$
\text { (31)-(35), (37), (46), (48)-(50), and (51)-(62). }
$$

The model (63) and (64) has linear, quadratic, and secondorder cone constraints, all of them convex. It also has continuous and integer variables. The commercial solver CPLEX [26] ensures global convergence for the proposed model.

\section{E. Mixed-Integer Linear Programing Model}

In order to compare the results of the MISOCP model for the OODN problem considering ESDs, an MILP model is presented below. Recall that (5), (23), and (37) are the nonlinear constraints of the model and (37) was obtained from (36) using conic relaxation. Instead of the presented approach, an MILP model can be obtained by linearizing these constraints. First, assume for (36) that the voltage magnitude interval $\left[\underline{V}^{2}, \bar{V}^{2}\right]$ is small, so that (65) is valid to approximate the left-hand side of (36)

$$
I_{i j, d}^{s q r} V_{j, d}^{s q r} \approx I_{i j, d}^{s q r}\left(V^{\mathrm{nom}}\right)^{2} \quad \forall i j \in \Omega_{b}, \forall d \in \Omega_{d} .
$$

Quadratic terms from (5), (23), and (36) can be approximated using piecewise linear functions

$$
\begin{gathered}
\left(P_{i, d}^{\mathrm{dg}}\right)^{2}+\left(Q_{i, d}^{\mathrm{dg}}\right)^{2} \approx f\left(P_{i, d}^{\mathrm{dg}}, \bar{S}_{i}^{\mathrm{dg}}, \Gamma\right)+f\left(Q_{i, d}^{\mathrm{dg}}, \bar{S}_{i}^{\mathrm{dg}}, \Gamma\right) \\
\forall i \in \Omega_{\mathrm{dg}}, \forall d \in \Omega_{d} \\
\left(P_{i, d}^{S}\right)^{2}+\left(Q_{i, d}^{S}\right)^{2} \approx f\left(P_{i, d}^{S}, \bar{S}_{i}^{S}, \Gamma\right)+f\left(Q_{i, d}^{S}, \bar{S}_{i}^{S}, \Gamma\right) \\
\forall i \in \Omega_{S}, \forall d \in \Omega_{d} \\
P_{i j, d}^{2}+Q_{i j, d}^{2} \approx f\left(P_{i j, d}, \bar{V} \bar{I}_{i j}, \Gamma\right)+f\left(Q_{i j, d}, \bar{V} \bar{I}_{i j}, \Gamma\right) \\
\forall i j \in \Omega_{b}, \forall d \in \Omega_{d} .
\end{gathered}
$$

The piecewise linear approximation function $f(y, \bar{y}, \Gamma)$ used in (66)-(68) is defined

$$
\begin{array}{ll}
f(y, \bar{y}, \Gamma)=\sum_{\gamma=1}^{\Gamma} \sigma_{y, \gamma} \delta_{y, \gamma} & \\
y=y^{+}+y^{-} & \\
y^{+}+y^{-}=\sum_{\gamma=1}^{\Gamma} \delta_{y, \gamma} & \\
0 \leq \delta_{y, \gamma} \leq \bar{y} / \Gamma & \forall \gamma=1, \ldots, \Gamma \\
\sigma_{y, \gamma}=(2 \gamma-1) \bar{y} / \Gamma & \forall \gamma=1, \ldots, \Gamma \\
y^{+}, y^{-} \geq 0 . &
\end{array}
$$

Equation (69) approximates the square value of variable $y$ by using variables $\delta_{y, \gamma}$ in order to discretize the absolute value of $y$. The parameter $\sigma_{y, \gamma}$ represents the slope of the $\gamma$ th line segment of the linearization method and is used to evaluate the contribution of $\delta_{y, \gamma}$ in each step of the discretization. The bound $\bar{y} / \Gamma$ in (72) and (73) represents the length of the $\Gamma$ discretized segments. $\delta_{y, \gamma}$ is a set of variables that denotes the length of the discretized segments, whose sum is equal to $|y|$ according to (71). $y^{+}$and $y^{-}$are nonnegative variables used to model $|y|$ and the parameter $\bar{y}$ is the maximum value of $y$.

The nonlinear constraints (5), (23), and (36) can be replaced by the linear constraints

$$
\begin{aligned}
& f\left(P_{i, d}^{\mathrm{dg}}, \bar{S}_{i}^{\mathrm{dg}}, \Gamma\right)+f\left(Q_{i, d}^{\mathrm{dg}}, \bar{S}_{i}^{\mathrm{dg}}, \Gamma\right) \leq\left(\bar{S}_{i}^{d g}\right)^{2} \\
& \forall i \in \Omega_{\mathrm{dg}}, \forall d \in \Omega_{d} \\
& f\left(P_{i, d}^{S}, \bar{S}_{i}^{S}, \Gamma\right)+f\left(Q_{i, d}^{S}, \bar{S}_{i}^{S}, \Gamma\right) \leq\left(\bar{S}_{i}^{S}\right)^{2} \\
& \forall i \in \Omega_{S}, \forall d \in \Omega_{d} \\
& I_{i j, d}^{s q r}\left(V^{\mathrm{nom}}\right)^{2}=f\left(P_{i j, d}, \bar{V} \bar{I}_{i j}, \Gamma\right)+ f\left(Q_{i j, d}, \bar{V} \bar{I}_{i j}, \Gamma\right) \\
& \forall i j \in \Omega_{b}, \forall d \in \Omega_{d} .
\end{aligned}
$$

Finally, the MILP model for the problem of OODN considering ESDs is given

$$
\min \sum_{d \in \Omega_{d}} \sum_{i \in \Omega_{S}} c_{d}^{S} \Delta t P_{i, d}^{S}+\sum_{d \in \Omega_{d}} \sum_{i \in \Omega_{\mathrm{dg}}} c_{d}^{\mathrm{dg}} \Delta t P_{i, d}^{\mathrm{dg}}
$$

subject to (6), (7), (8), (10), (11), (17)-(20), (22),

(31)-(35), (46), (48)-(50), (51)-(62), and (75)-(77). 


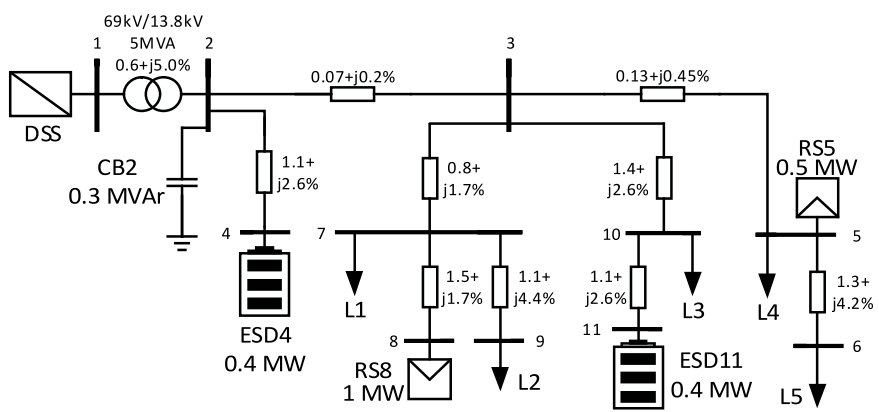

Fig. 6. Eleven-node test system of Levron et al. [14].

Notice that the linearization of the quadratic and conic constraints increases the number of continuous variables, but the number of integer variables remains unchanged. Also, greater values of $\Gamma$ improve the fitting of the piecewise linear approximation function, but also increases the size of the problem.

\section{TESTS AND RESUlts}

An 11-node test system and a real 42-node system were used to demonstrate the performance and robustness of the proposed methodologies. For both systems, $\Delta t=0.5 \mathrm{~h}, \underline{V}=0.95$ p.u., and $\bar{V}=1.05$ p.u. The proposed MISOCP and MILP models, given by (63)-(64) and (78)-(79), respectively, were implemented in the mathematical programing language AMPL [27] and solved using the commercial solver for optimization problems CPLEX (version 12.6.0.0, 64 bits using default settings) on a computer with a $3.40 \mathrm{GHz}$ Intel core i7 processor and $16 \mathrm{~GB}$ of RAM. Complete results are shown for the MISOCP model, while only numerical results are shown for the MILP model for comparison.

\section{A. 11-Node Test System}

In order to validate the proposed model, it was first analyzed in a slightly modified version of the microgrid proposed by Levron et al. [14]. Fig. 6 shows the test system containing two photovoltaic RSs with power peaks of 1 and $0.5 \mathrm{MW}$ at nodes 8 and 5, respectively; two ESDs at nodes 4 and 11 with $0.4 \mathrm{MWh}$ capacities each and a self-discharge rate of $\beta_{i}^{s d}=0.021$; a CB of 0.3 MVAr at node 2; and five loads.

The objective was to minimize the cost of energy purchase from node 1. Impedances are in percent with a base (power and voltage magnitude) equal to the transformer's nominal ratings. It was also assumed that both ESDs had the same value of efficiency $\eta_{i}^{s d+}=\eta_{i}^{s d-}=0.95$. The maximum daily change in operation of the ESDs was equal to 2 .

The cost of energy $c_{d}^{S}$, the active and reactive total load power, and the active power of photovoltaic RSs (shown in Fig. 7) were the same as those utilized in [14]. They were generated over a 72 -h period. Seven cases were analyzed with different parameter values. For each case, the values of the modified parameters were specified, while all other values remained the same as previously cited. Complete results are shown for Cases A1 and A2, while for Cases A3-A7 the

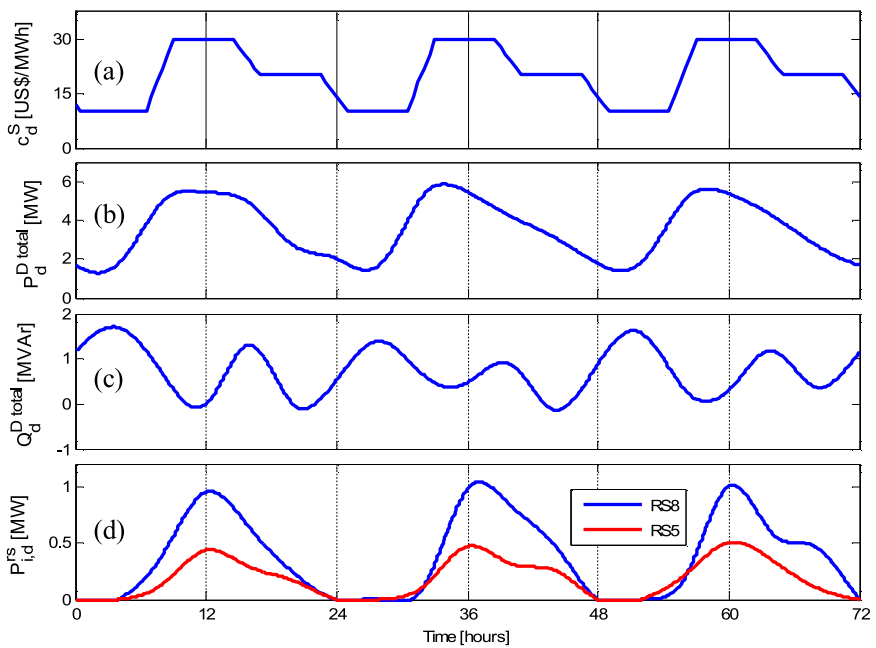

Fig. 7. Eleven-node test system of Levron et al. [14]. (a) Cost of energy purchase from node 1. (b) Total active load power. (c) Total reactive load power. (d) Active power of photovoltaic RSs.

TABLE I

Results for CASES Using THE 11-Node Test System

\begin{tabular}{|c|c|c|c|c|c|c|}
\hline \multirow{2}{*}{$\begin{array}{r}\text { Test } \\
\text { Case }\end{array}$} & $\begin{array}{c}\text { Total Energy Losses in } \\
\text { Lines [kWh] }\end{array}$ & $\begin{array}{r}\text { Total Energy Losses in } \\
\text { ESDs [kWh] }\end{array}$ & \multicolumn{2}{|c|}{ Operation Cost [US\$] } \\
\cline { 2 - 7 } & MISOCP & MILP & MISOCP & MILP & MISOCP & MILP \\
\hline A1 & 2039.10 & 2039.10 & - & - & 4801.99 & 4801.99 \\
\hline A2 & 2047.68 & 2047.79 & 385.68 & 385.65 & 4762.65 & 4762.65 \\
\hline A3 & 2056.12 & 2056.18 & 379.00 & 378.95 & 4761.00 & 4761.00 \\
\hline A4 & 2021.45 & 2024.06 & 0.00 & 0.00 & 4753.25 & 4753.28 \\
\hline A5 & 2087.26 & 2089.79 & 777.28 & 775.09 & 4720.65 & 4720.66 \\
\hline A6 & 2043.00 & 2043.00 & 126.32 & 126.32 & 4788.29 & 4788.29 \\
\hline A7 & 2058.02 & 2058.02 & 511.58 & 511.58 & 4763.42 & 4763.42 \\
\hline
\end{tabular}

results are reported in Table I. The cases are described as follows.

Case A1: Without ESDs in the system and considering a transformer with increased capacity: $\bar{S}_{1}^{S}=10$ MVA.

Case A2: With both ESDs in the system and considering the original transformer capacity: $\bar{S}_{1}^{S}=5$ MVA.

In order to analyze the influence of different characteristics of the ESDs in the system, the following cases consider a transformer with increased capacity: $\bar{S}_{1}^{S}=10$ MVA.

Case A3: With the ESDs and considering a transformer with increased capacity $\left(\bar{S}_{1}^{S}=10 \mathrm{MVA}\right)$.

Case A4: Given ideal ESDs, i.e., $\eta_{i}^{s d+}=\eta_{i}^{s d-}=1$ and $\beta_{i}^{s d}=0$, in this case, the ESDs will not present losses.

Case A5: ESDs at nodes 4 and 11 with 0.8 MWh each. This case considers ESDs with twice the capacity of the ESDs of Case A3.

Case A6: Only two changes in operation for each ESD during the analyzed period.

Case A7: Double the self-discharge for each ESD, i.e., $\beta_{i}^{s d}=0.042$.

The optimal solutions for Cases A1 and A2 are shown in Fig. 8. Fig. 8(a) shows the total energy stored by the ESDs in Case A2. As expected, the energy was stored when the 


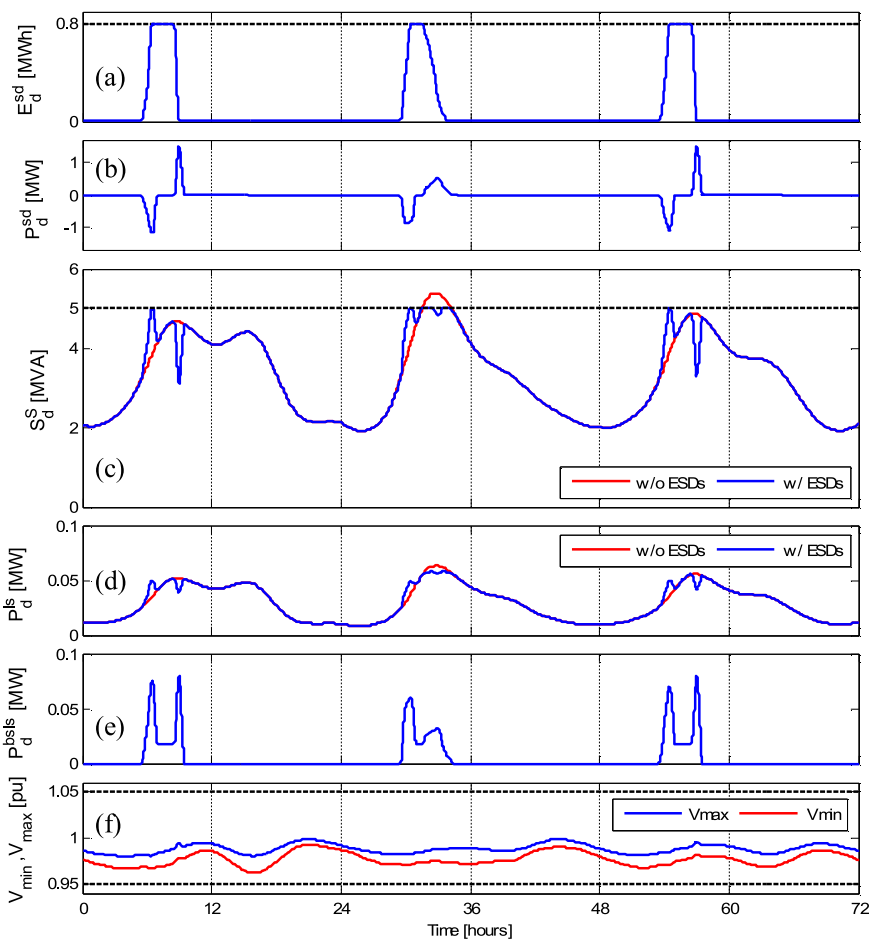

Fig. 8. Optimal operation of the 11-node test system. (a) Total stored energy (Case A2). (b) Total active power extracted and injected by the ESDs (Case A2). (c) Apparent power generation at node $1, S_{d}^{S}$, without and with the ESDs in the system (Cases A1 and A2). (d) Active power losses in the lines without and with the ESDs in the system (Cases A1 and A2). (e) Active power losses in the ESDs (Case A2). (f) Voltage profile on the load nodes with the highest and lowest voltages during the operation period, Cases A1 and A2 (same profiles).

energy cost was low and injected into the DN when the energy cost was high. Moreover, it is easy to see the relationship between Fig. 8(a) and (b): when power is extracted from the system [negative values in Fig. 8(b) between 5:30 and 6:30 A.M. on all three days of Case A2], the amount of stored energy increases, meeting the maximum value of $0.8 \mathrm{MWh}$; when power is injected into the system [positive values in Fig. 8(b) between 8:30 and 9:30 A.M. on days 1 and 3, and between 7:30 and 10:00 A.M. on day 2], the stored energy decreases. Because the maximum number of daily changes in operation for the ESD is two, there is a total of six periods of operation for the ESDs-three of them extracting and three of them injecting power. Fig. 8(c) shows the apparent power generation of the DSS with (Case A2) and without (Case A1) the ESDs in the system. Without the ESDs, the system operation violates the capacity of the transformer considered in Case A2, and the problem is infeasible (for Case A2). Fig. 8(d) and (e) shows the power losses in the lines (for Cases A1 and A2) and in the ESDs (Case A2), respectively. Note that when the ESDs are extracting/injecting power in the system, the losses increase. When they are charged, due to self-discharge, there is a small amount of loss. Fig. 8(f) shows that all voltages in the system are within their limits (Case A2). Constraint (37) was also active for all branches in both cases.

The results for Cases A1-A7 are shown in Table I, which contains information about total energy losses in lines and ESDs, and the cost of energy purchase

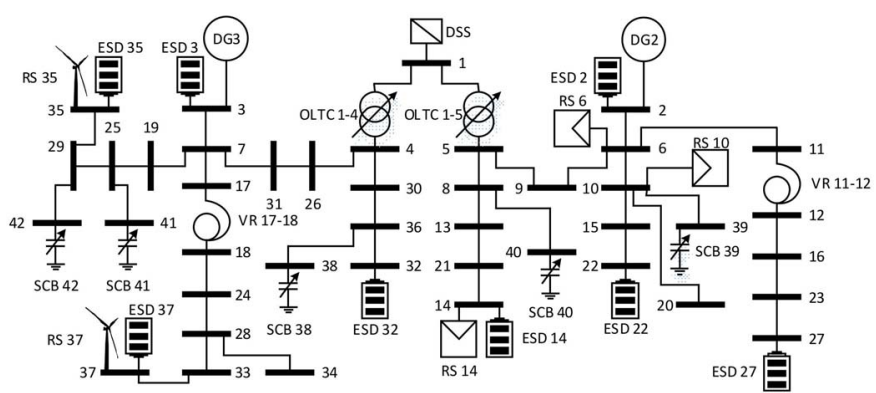

Fig. 9. Forty-two-node real system.

(value of the objective function). Comparing the results of Cases A1 and A2, as shown in Table I, an economy of energy purchase of $0.82 \%$ is obtained when the ESDs are present in the system. With a transformer with increased capacity (Case A3), the economy is $0.85 \%$, when compared to Case A1. If the ESDs were ideal (Case A4), the economy would be $1.02 \%$. With ESDs possessing twice the capacity (Case A5), the economy is $1.69 \%$. Limiting the changes, the operation with two changes in the period (Case A6) reduces the economy to $0.29 \%$. Finally, doubling the self-discharge rate of both ESDs (Case A7) reduces the economy to $0.80 \%$. Also, note that the results obtained with the MISOCP and MILP models are almost identical.

The minimum and the maximum voltage magnitudes in the system (on load nodes) for all cases were 0.9621 and 0.9985 p.u., respectively.

\section{B. 42-Node Real System}

Fig. 9 shows the second system analyzed. It is a 42-node real DN [28] containing two OLTCs with five tap positions $(-2 ;-1 ; 0 ;+1 ;+2)$ and a regulation of $2.5 \%$ per tap ( $\pm 5 \%$ of maximum regulation); two VRs with 33 tap positions $(-16 ;-15 ;-14 ; \ldots ; 0 ;+1 ;+2 ; \ldots ;+16)$ and a regulation of $0.625 \%$ per tap ( $\pm 10 \%$ of maximum regulation); five SCBs of $480 \mathrm{kvar}$ with four units (120 kvar/unit) at nodes 38, 39, and 40, and of $1200 \mathrm{kvar}$ (300 kvar/unit) at nodes 41 and 42; two biomass dispatchable DGs, both with a nominal capacity of 1 MVA and capacitive and inductive minimum power factors of 1 and 0.95 , respectively; two wind turbines, three photovoltaic generators, and eight ESDs with a capacity of $0.8 \mathrm{MWh}$ each. It was assumed that $\bar{\Delta}_{i}^{\text {scb }}=4$ per day for all SCBs and $\bar{\Delta}_{i j}^{\mathrm{vr}}=4$ for all OLTCs, and $\bar{\Delta}_{i j}^{\mathrm{vr}}=11$ for all VRs. The objective is to minimize the cost of energy purchased from the DSS at node 1 and from dispatchable DGs 2 and 3. The voltage at the DSS is $13.8 \mathrm{kV}$, and its capacity is 50 MVA. Fig. 10(a) shows the cost of energy, $c_{d}^{S}$. Figs. 10(b)-(c) show the active and reactive total load power. Figs. 10(d)-(e) show the active power of RSs. These were generated over a 72-h period.

We also assumed an efficiency of $\eta_{i}^{s d+}=\eta_{i}^{s d-}=0.95$, $\beta_{i}^{s d}=0.01, \underline{P}_{i}^{s d+}=\underline{P}_{i}^{s d-}=\underline{E}_{i}^{s d}=0$, and $\bar{P}_{i, d}^{s d+}=\bar{P}_{i, d}^{s d-}=$ $800 \mathrm{~kW}$ for all ESDs. Two cases were analyzed: without the presence of the ESDs in the system (Case B1) and considering the ESDs (Case B2). Results for both cases are presented.

Case B1: The global optimal solution for the model (63) and (64) for the 42-node DN without the 

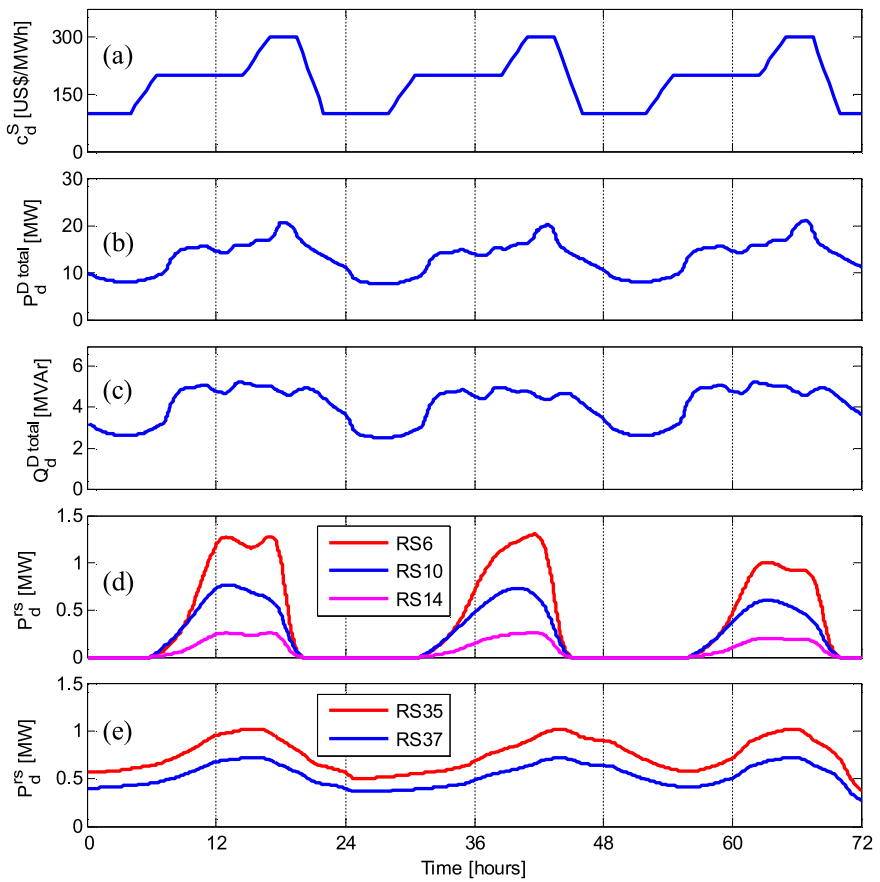

Fig. 10. Forty-two-node real system. (a) Cost of energy purchase from node 1 and dispatchable DGs. (b) Total active load power. (c) Total reactive load power. (d) Active power of photovoltaic RSs. (e) Active power of wind turbines RSs.

ESDs is shown in Fig. 11. Constraint (37) was also active for all branches. Fig. 11 shows the sum of reactive power injected by all the SCBs, the tap movements of the VRs, the total active and reactive power injected by the dispatchable DGs, and the voltage profile of the system. Fig. 11(a) shows that more units of SCBs were connected when the load was heavy. The taps of both OLTCs remained at position +2 for all load levels. Note that the voltages were always within their limits.

Case B2: Figs. 12 and 13 show the optimal solution for the 42-node DN considering the ESDs. Constraint (37) was active for all branches. Figs. 11 and 12 contain the same information, from which one can draw the same conclusions. Fig. 13 shows the total energy stored in the ESDs, total injected and extracted power by the ESDs, the apparent power injected by the DSS, the active power losses in the lines, the active power losses in the ESDs, and the voltage profile of the system. Again, as in the previous test system, energy was stored when the energy price was low and injected into the system when energy was expensive.

Due to the self-discharge rate, there was a small decrease in stored energy over the three days, as can be seen in Fig. 13(a). Likewise, all voltages in the system were within their limits, as can be seen in Fig. 13(f). In Fig. 13(a), the numbered arrows indicate the phases of operation of the ESDs. During intervals 1 and 2, the ESDs were discharged. From 2, they started charging, reaching full charge at 3 . In intervals 3 and 4 , the ESDs neither extracted nor injected power in the system; so, due to self-discharge effect, they lost energy. At 4, they began extracting energy from the system, acquiring again full charge. During intervals 5 and 6, power was injected into the system, so the ESDs discharged. Afterwards, they remained uncharged
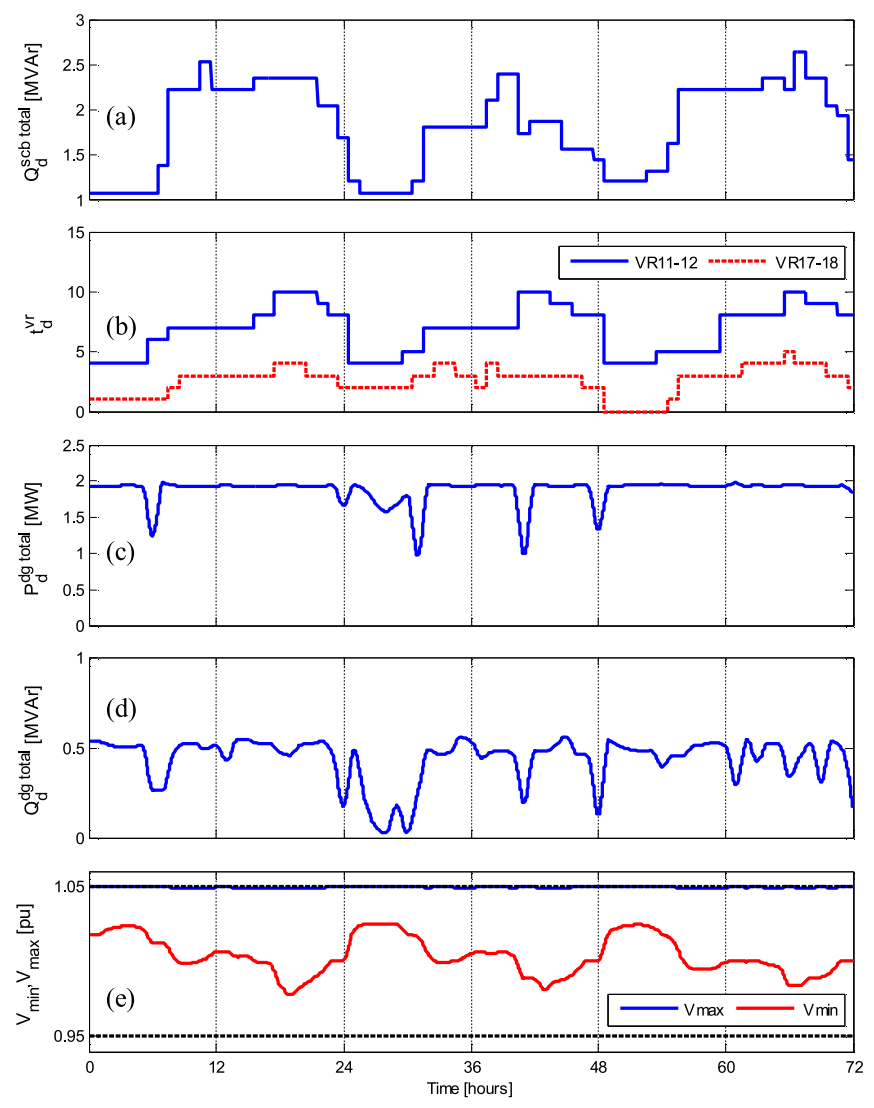

Fig. 11. Optimal operation of the 42-node real system without ESDs (Case B1). (a) Total reactive power injected by the SCBs. (b) Tap movements of the VRs. (c) Total active power injected by the dispatchable DGs. (d) Total reactive power injected by the dispatchable DGs. (e) Voltage profile of the load nodes with the highest and lowest voltage during the operation period.

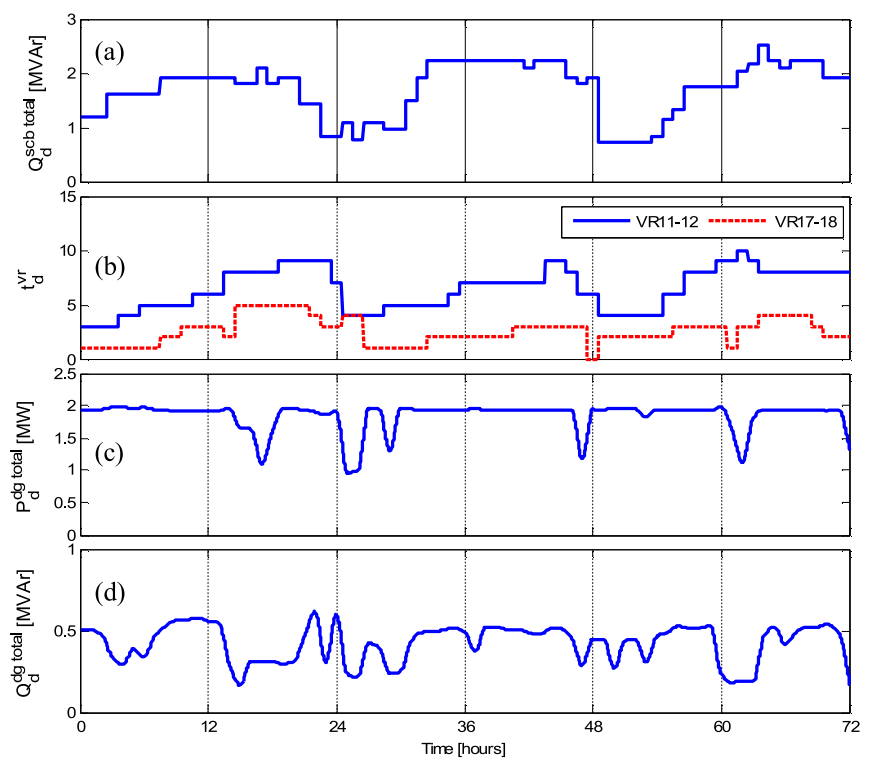

Fig. 12. Optimal operation of the 42-node real system with ESDs (Case B2). (a) Total reactive power injected by the SCBs. (b) Tap movements of the VRs. (c) Total active power injected by the dispatchable DGs. (d) Total reactive power injected by the dispatchable DGs.

during the periods 6 and 7; subsequently, they repeated a similar cycle. From Fig. 13(a), it can be seen that this cycle was similar for all three days. 

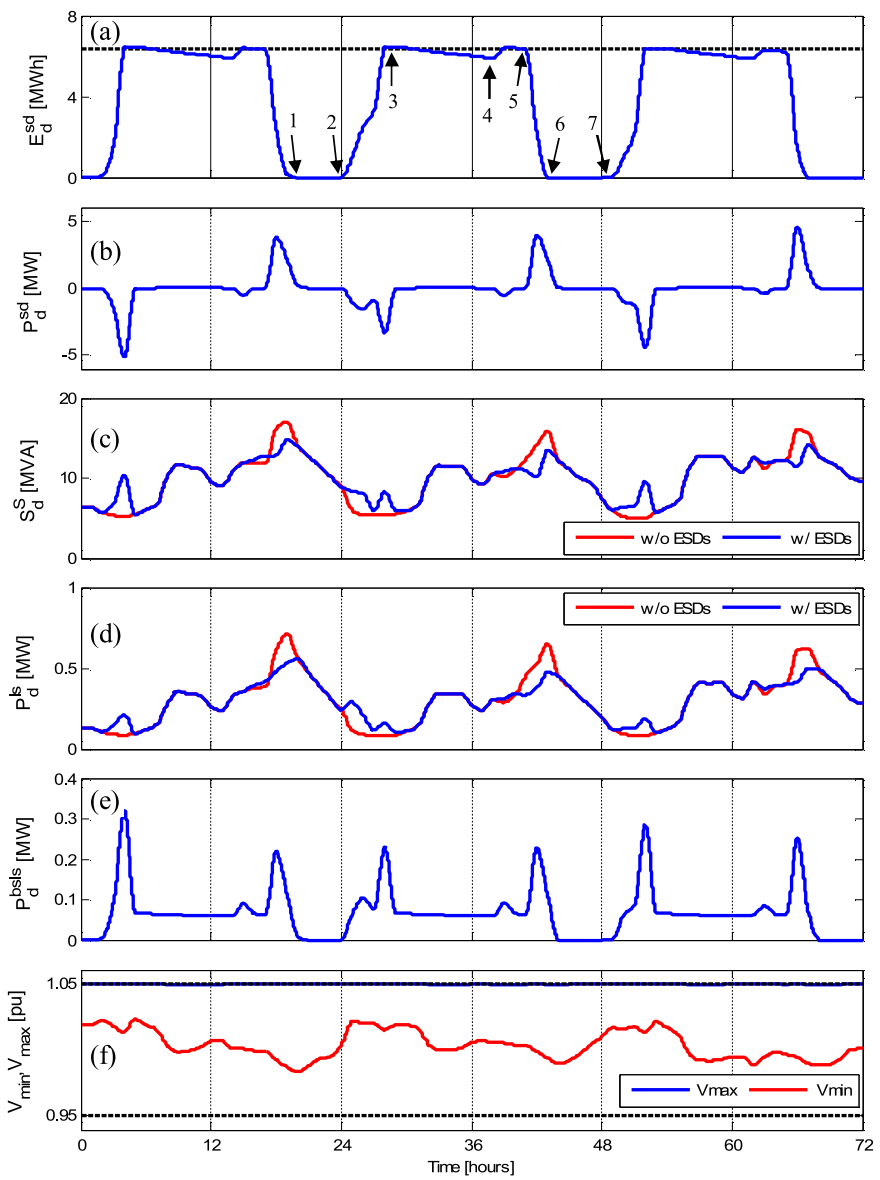

Fig. 13. Optimal operation of the 42-node real system with ESDs. (a) Total stored energy (Case B2). (b) Total active power extracted and injected by the ESDs (Case B2). (c) Apparent power generated at node 1, $S_{d}^{S}$, without and with the ESDs in the system (Cases B1 and B2). (d) Active power losses in the lines without and with the ESDs in the system (Cases B1 and B2). (e) Active power losses in the ESDs (Case B2). (f) Voltage profile of the load nodes with the highest and lowest voltage during the operation period (Case B2).

TABLE II

Results For CAses Using The 42-Node ReAl System

\begin{tabular}{|c|c|c|c|c|c|c|c|}
\hline $\begin{array}{c}\text { Test } \\
\text { Case }\end{array}$ & $\begin{array}{c}\text { Total Energy Loss- } \\
\text { es in Lines [kWh] }\end{array}$ & $\begin{array}{r}\text { Total Energy } \\
\text { Losses in ESDs } \\
{[\mathrm{kWh}]}\end{array}$ & \multicolumn{2}{|c|}{ Operation Cost [U\$\$] } & $\begin{array}{c}\text { Cost } \\
\text { Difference } \\
{[\%]}\end{array}$ \\
\cline { 2 - 7 } & MISOCP & MILP & MISOCP & MILP & MISOCP & MILP & \\
\hline B1 & 21681.27 & 23445.82 & - & - & 165961.28 & 166341.44 & 0.23 \\
\hline B2 & 21610.85 & 23167.84 & 4870.27 & 4819.64 & 162882.78 & 163228.96 & 0.21 \\
\hline
\end{tabular}

It must be clarified that the ESDs do not necessarily inject or extract power at the same time, i.e., at any given time, some ESDs may inject power, while others extract power. The mathematical model defines the optimal operation for each device. Further results for Cases B1 and B2 are shown in Table II. The values of total energy losses in lines and ESDs, and the cost of energy purchase are presented.

The results in Table II show that an economy in energy purchase cost of $1.85 \%$ is obtained with the ESDs in the system (using the MISOCP model). The operation cost for the results of the MISOCP model and the MILP model for this case are also close. Finally, it is emphasized that the model is flexible since the maximum number of changes in operation for
TABLE III

Computational Time Needed to Solve the Models FOR CASES Using THe 11-Node Test System

\begin{tabular}{|c|c|c|c|c|c|c|c|}
\hline \multirow{2}{*}{ Time $[\mathrm{s}]$} & \multicolumn{7}{|c|}{ Test Case } \\
\cline { 2 - 8 } & $\mathrm{A} 1$ & $\mathrm{~A} 2$ & $\mathrm{~A} 3$ & $\mathrm{~A} 4$ & $\mathrm{~A} 5$ & $\mathrm{~A} 6$ & $\mathrm{~A} 7$ \\
\hline MISOCP & - & 1.56 & 1.32 & 10.09 & 1.51 & 16.16 & 1.18 \\
\hline MILP & - & 1.42 & 1.01 & 1.85 & 1.11 & 34.98 & 0.89 \\
\hline
\end{tabular}

TABLE IV

Computational Time NeEded to

SOLVE THE MODELS FOR CASES

USING THE 42-NODE

REAL SYSTEM

\begin{tabular}{|c|c|c|}
\hline \multirow{2}{*}{ Time [s] } & \multicolumn{2}{|c|}{ Test Case } \\
\cline { 2 - 3 } & $\mathrm{B} 1$ & $\mathrm{~B} 2$ \\
\hline MISOCP & 385.03 & 420.87 \\
\hline MILP & 141.90 & 197.34 \\
\hline
\end{tabular}

every piece of equipment is adjustable. Thus, if a solution that requires all controls to be adjusted only once over a period is desired, the proposed model can accommodate it.

\section{Computational Performance}

Below, further information is presented regarding the time needed to solve the problems presented in this paper, considering both MISOCP and MILP models. The computational time needed to solve Cases A2-A7 using the 11-node system is shown in Table III. Since Case A1 was a simple power flow solution for each load level, the time to solve it has been omitted. Table IV shows the time needed to solve Cases B1 and B2 for the 42-node system.

The results shown in Tables I-IV indicate that the MISOCP makes it possible to find the optimal solution of the problem in a reasonable amount of time. However, an MILP is an interesting alternative if a good quality solution is needed in a shorter amount of time. For the tests carried out, the time expended by the MILP was about half that required by the MISOCP; in real applications, this difference in the processing time can be taken into account.

\section{CONCLUSION}

This paper presented MISOCP and MILP models for solving the problem of OODNs considering ESDs. The proposed models considered as control variables the generation of active and reactive power of dispatchable DGs, the number of units in operation in each SCB, the tap position of the VRs and OLTCs, and the operation state of the ESDs.

The objective was to minimize the total cost of energy purchase from the DSS and the dispatchable DGs. The steadystate operation of the radial DN was modeled through the use of linear and second-order conic expressions. The use of an MISOCP model guarantees convergence to optimality using classical optimization techniques.

An 11-node test system and a 42-node real distribution system were used to demonstrate the accuracy of the mathematical models, as well as the efficiency of the proposed solution 
techniques. The results showed that it is possible to determine the OODNs with ESDs using a flexible, realistic, and precise MISOCP model. The approximated MILP model provides good-quality solutions with shorter computational time.

\section{ACKNOWLEDGMENT}

The authors would like to thank Y. Levron for his help in providing the test system data and results.

\section{REFERENCES}

[1] A. B. M. Shawkat Ali, Ed., Smart Grids: Opportunities, Developments, and Trends, Green Energy and Technology. New York, NY, USA: Springer, 2013.

[2] T. Senjyu, Y. Miyazato, A. Yona, N. Urasaki, and T. Funabashi, "Optimal distribution voltage control and coordination with distributed generation," IEEE Trans. Power Del., vol. 23, no. 2, pp. 1236-1242, Apr. 2008

[3] A. Augugliaro, L. Dusonchet, S. Favuzza, and E. R. Sanseverino, "Voltage regulation and power losses minimization in automated distribution networks by an evolutionary multiobjective approach," IEEE Trans. Power Syst., vol. 19, no. 3, pp. 1516-1527, Aug. 2004.

[4] Y. P. Agalgaonkar, B. C. Pal, and R. A. Jabr, "Distribution voltage control considering the impact of PV generation on tap changers and autonomous regulators," IEEE Trans. Power Syst., vol. 29, no. 1, pp. 182-192, Jan. 2014.

[5] R. A. Jabr, "Minimum loss operation of distribution networks with photovoltaic generation," IET Renew. Power Gen., vol. 8, no. 1, pp. 33-44, Jan. 2014

[6] R. R. Gonçalves, R. P. Alves, J. F. Franco, and M. J. Rider, "Operation planning of electrical distribution systems using a mixed integer linear model," J. Control Autom. Elect. Syst., vol. 24, no. 5, pp. 668-679, Jun. 2013.

[7] R. A. Araujo, P. C. M. Meira, and M. C. de Almeida, "Algorithms for operation planning of electric distribution networks," J. Control Autom. Elect. Syst., vol. 24, no. 3, pp. 377-387, Jun. 2013.

[8] F. A. Chacra, P. Bastard, G. Fleury, and R. Clavreul, "Impact of energy storage costs on economical performance in a distribution substation," IEEE Trans. Power Syst., vol. 20, no. 2, pp. 684-691, May 2005.

[9] Y. Levron and D. Shmilovitz, "Power systems' optimal peak-shaving applying secondary storage," Elect. Power Syst. Res., vol. 89, pp. 80-84, Aug. 2012.

[10] Y. Levron and D. Shmilovitz, "Optimal power management in fueled systems with finite storage capacity," IEEE Trans. Circuits Syst. I, Reg. Papers, vol. 57, no. 8, pp. 2221-2231, Aug. 2010.

[11] J. P. Barton and D. G. Infield, "Energy storage and its use with intermittent renewable energy," IEEE Trans. Energy Convers., vol. 19, no. 2, pp. 441-448, Jun. 2004.

[12] T. K. A. Brekken et al., "Optimal energy storage sizing and control for wind power applications," IEEE Trans. Sustain. Energy, vol. 2, no. 1, pp. 69-77, Jan. 2011.

[13] P. Poonpun and W.T. Jewell, "Analysis of the cost per kilowatt hour to store electricity," IEEE Trans. Energy Convers., vol. 23, no. 2, pp. 529-534, Jun. 2008.

[14] Y. Levron, J. M. Guerrero, and Y. Beck, "Optimal power flow in microgrids with energy storage," IEEE Trans. Power Syst., vol. 28, no. 3, pp. 3226-3234, Aug. 2013.

[15] A. Gabash and P. Li, "Active-reactive optimal power flow in distribution networks with embedded generation and battery storage," IEEE Trans. Power Syst., vol. 27, no. 4, pp. 2026-2035, Nov. 2012.

[16] A. Gabash and P. Li, "Flexible optimal operation of battery storage systems for energy supply networks," IEEE Trans. Power Syst., vol. 28, no. 3, pp. 2788-2797, Aug. 2013.

[17] S. Gill, I. Kockar, and G. W. Ault, "Dynamic optimal power flow for active distribution networks," IEEE Trans. Power Syst., vol. 29, no. 1, pp. 121-131, Jan. 2014.

[18] D. Pozo, J. Contreras, and E. E. Sauma, "Unit commitment with ideal and generic energy storage units," IEEE Trans. Power Syst., vol. 29, no. 6, pp. 2974-2984, Nov. 2014.

[19] F. Glover and G. Kochenberger, Handbook of Metaheuristics. Norwell, MA, USA: Kluwer Academic, 2003.
[20] J. F. Franco, M. J. Rider, M. Lavorato, and R. Romero, "Optimal conductor size selection and reconductoring in radial distribution systems using a mixed-integer LP approach," IEEE Trans. Power Syst., vol. 28, no. 1, pp. 10-20, Feb. 2013.

[21] D. Shirmohammadi, H. W. Hong, A. Semlyen, and G. X. Luo, "A compensation-based power flow method for weakly meshed distribution and transmission networks," IEEE Trans. Power Syst., vol. 3, no. 2, pp. 753-762, May 1988.

[22] R. G. Cespedes, "New method for the analysis of distribution networks," IEEE Trans. Power Del., vol. 5, no. 1, pp. 391-396, Jan. 1990.

[23] R. A. Jabr, "Radial distribution load flow using conic programming," IEEE Trans. Power Syst., vol. 21, no. 3, pp. 1458-1459, Aug. 2006.

[24] J. F. Franco, M. J. Rider, and R. Romero, "A mixed-integer quadraticallyconstrained programming model for the distribution system expansion planning," Int. J. Elect. Power Energy Syst., vol. 62, pp. 265-272, Nov. 2014.

[25] M. Farivar and S. H. Low, "Branch flow model: Relaxations and convexification-Part I," IEEE Trans. Power Syst., vol. 28, no. 3 , pp. 2554-2564, Aug. 2013.

[26] (Apr. 10, 2014). IBM ILOG CPLEX. IBM. [Online]. Available: http://www-01.ibm.com/software/integration/optimization/ cplex-optimization-studio

[27] R. Fourer, D. M. Gay, and B. W. Kernighan, AMPL: A Modeling Language for Mathematical Programming. 2nd ed. Pacific Grove, CA, USA: Brooks/Cole-Thomson Learning, 2003.

[28] LaPSEE Power System Test Cases Repository. (2014, Oct. 27). [Online]. Available: http://www.feis.unesp.br/\#!/lapsee

Leonardo H. Macedo (S'14) received the B.Sc. and M.Sc. degrees from the Universidade Estadual Paulista, Ilha Solteira, São Paulo, Brazil, in 2012 and 2015 , respectively, where he is currently pursuing the Ph.D. degree, all in electrical engineering.

His current research interests include the development of methodologies for the optimization, planning, and control of electrical power systems.

John F. Franco (S'11-M'13) received the B.Sc. and M.Sc. degrees from the Universidad Tecnológica de Pereira, Pereira, Colombia, in 2004 and 2006, respectively, and the Ph.D. degree from the Universidade Estadual Paulista, Ilha Solteira, São Paulo, Brazil, in 2012, all in electrical engineering.

$\mathrm{He}$ is currently a Post-Doctoral Research Fellow with the Universidade Estadual Paulista. His current research interests include the development of methodologies for the optimization and planning of distribution systems.

Marcos J. Rider (S'97-M'06) received the B.Sc. (Hons.) and P.E. degrees from the National University of Engineering, Lima, Perú, in 1999 and 2000, respectively; the M.Sc. degree from the Federal University of Maranhão, Maranhão, Brazil, in 2002; and the Ph.D. degree from the University of Campinas, Campinas, Brazil, in 2006, all in electrical engineering.

$\mathrm{He}$ is currently a Professor with the Electrical Engineering Department, Universidade Estadual Paulista, Ilha Solteira, Brazil. His current research interests include the development of methodologies for the optimization, planning, and control of electrical power systems and applications of artificial intelligence in power systems.

Rubén Romero (M'93-SM'08) received the B.Sc. and P.E. degrees from the National University of Engineering, Lima, Perú, in 1978 and 1984, respectively, and the M.Sc. and Ph.D. degrees from the University of Campinas, Campinas, Brazil, in 1990 and 1993, respectively, all in electrical engineering.

$\mathrm{He}$ is currently a Professor with the Electrical Engineering Department, Universidade Estadual Paulista, Ilha Solteira, Brazil. His current research interests include the area of electrical power systems planning. 